\title{
Erectile function and late-onset hypogonadism symptoms related to lower urinary tract symptom severity in elderly men
}

\author{
Ozan Bozkurt ${ }^{1}$, Deniz Bolat ${ }^{2}$, Omer Demir ${ }^{1}$, Oktay Ucer $^{3}$, Ali Şahin $^{4}$, Burak Ozcift ${ }^{5}$, Abdulkadir Pektaş $^{6}$, \\ Tahir Turan ${ }^{7}$, Bilal H Gümüş ${ }^{3}$, Ertan Can $^{8}$, Ahmet Bolukbasi ${ }^{5}$, Haluk Erol ${ }^{9}$ and Adil Esen ${ }^{1}$
}

The aim of this study was to evaluate the relationship between lower urinary tract symptoms (LUTSs), erectile dysfunction (ED) and symptomatic late-onset hypogonadism (SLOH) in ageing men in the Aegean region of Turkey. Five hundred consecutive patients $>40$ years old who had been in a steady sexual relationship for the past 6 months and were admitted to one of six urology clinics were included in the study. Serum prostate-specific antigen and testosterone levels and urinary flow rates were measured. All patients filled out the International Prostate Symptom Score and Quality of Life (IPSS-QoL), International Index of Erectile Function (IIEF) and Aging Males' Symptoms (AMS) scale forms. Of the patients, $23.9 \%$ had mild LUTSs, $53.3 \%$ had moderate LUTSs and $22.8 \%$ had severe LUTSs. The total testosterone level did not differ between groups. Additionally, $69.6 \%$ had ED. The presence of impotence increased with increasing LUTS severity. Symptomatic late-onset hypogonadism (AMS $>27$ ) was observed in $71.2 \%$ of the patients. The prevalence of severe hypogonadism symptoms increased with the IPSS scores. A correlation analysis revealed that all three questionnaire scores were significantly correlated. In conclusion, LUTS severity is an age-independent risk factor for ED and SLOH. LUTS severity and SLOH symptoms appear to have a strong link that requires etiological and biological clarification in future studies. Asian Journal of Andrology (2013) 15, 785-789; doi:10.1038/aja.2013.44; published online 1 July 2013

Keywords: ageing male; erectile dysfunction; lower urinary tract symptoms; symptomatic late-onset hypogonadism; symptom scores; testosterone

\section{INTRODUCTION}

There has been dramatic worldwide increase in the elderly population during the twentieth century. Thus, in recent years, the symptoms and complaints of ageing men have found increasing scientific attention. Increasing age is associated with various physiologic changes in men such as decreased sexual function and increased voiding problems. Lower urinary tract symptoms (LUTSs), erectile dysfunction (ED) and symptomatic late-onset hypogonadism (SLOH) are the most common urological complaints in elderly men.

There are various large-scale community-based studies showing the association between LUTSs and ED. ${ }^{1-4}$ The following theories have been proposed for the possible pathophysiological links: the nitric oxide synthase/nitric oxide theory; the autonomic hyperactivity and metabolic syndrome hypothesis; the Rho-kinase activation/endothelin pathway; and pelvic atherosclerosis. ${ }^{5}$ However, it seems difficult to explain the relationship between these two conditions with just one of the above-mentioned theories. All of these theories may contribute to the development of these conditions in different stages.
SLOH is a condition composed of different physiological, somatic, vegetative and sexual complaints due to decreasing androgen levels in elderly men. The prevalence of hypogonadism in men ranges from $6.0 \%$ to $12.3 \%$ between the ages of 40 and 69 years, and it is estimated that 2.4 million men in the United States have androgen deficiency. ${ }^{6}$ The association between LUTSs and SLOH might be important because both conditions affect ageing men and the management of one condition may have an improving effect on the other. However, in these studies, only a hormonal evaluation was performed for SLOH, not a symptomatic evaluation.

In this study, we evaluated the relationship between LUTSs, ED and SLOH in patients presenting with LUTSs in the Aegean region of Turkey. Validated questionnaires were used for this purpose.

\section{MATERIALS AND METHODS}

Participants

Five hundred consecutive patients aged $>40$ years who had been in a steady sexual relationship for the past 6 months and were admitted to a urology clinic with LUTSs were included. All patients gave their

${ }^{1}$ Department of Urology, Dokuz Eylul University, Faculty of Medicine, Izmir 35340, Turkey; ${ }^{2}$ Division of Urology, Doc. Dr. Yasar Eryilmaz Dogubayazit State Hospital, Agri 04900, Turkey; ${ }^{3}$ Department of Urology, Celal Bayar University, Manisa 45040, Turkey; ${ }^{4}$ Department of Urology, Sifa University, Izmir 35230 , Turkey; ${ }^{5}$ Clinic of Urology, Ataturk Training and Research Hospital, Izmir 35965, Turkey; ${ }^{6}$ Division of Urology, Adiyaman 82. Y1I State Hospital, Adiyaman 02040, Turkey; ${ }^{7}$ Department of Urology, Pamukkale University, Faculty of Medicine, Denizli 20020, Turkey; ${ }^{8}$ Clinic of Urology, Tepecik Training and Research Hospital, Izmir 35120, Turkey and ${ }^{9}$ Department of Urology, Adnan Menderes University, Faculty of Medicine, Aydin 09100, Turkey

Correspondence: Dr O Bozkurt (ozan.bozkurt@deu.edu.tr)

Received: 4 January 2013; Revised: 4 March 2013; Accepted: 17 March 2013; Published online: 1 July 2013 
Table 1 Comparison of the evaluated parameters between the three groups

\begin{tabular}{|c|c|c|c|c|}
\hline & \multicolumn{3}{|c|}{ LUTS severity } & $\mathrm{P}$ \\
\hline$N(\%)$ & $118(24.0)$ & $262(53.2)$ & $112(22.8)$ & \\
\hline Prevalence of ED (\%) & 60.7 & 69.9 & 75.7 & 0.027 \\
\hline IIEF-EF & $20.7 \pm 8.6$ & $18.4 \pm 9.2$ & $15.7 \pm 9.8$ & $<0.001^{\mathrm{b}}$ \\
\hline $\operatorname{MFR}\left(\mathrm{ml} \mathrm{s}^{-1}\right)$ & $19.5 \pm 16.9$ & $14.6 \pm 6.9$ & $13.0 \pm 11.2$ & $<0.001^{a}$ \\
\hline Testosterone $\left(\mathrm{ng} \mathrm{ml}^{-1}\right)$ & $464.5 \pm 151.3$ & $468.5 \pm 161.8$ & $456.0 \pm 153.9$ & 0.795 \\
\hline
\end{tabular}

Abbreviations: ED, erectile dysfunction; IIEF-EF, International Index of Erectile Function-Erectile Function; IPSS-QoL, International Prostate Symptom Score-Quality of Life; LUTS, lower urinary tract symptom; MFR, maximum flow rate; PSA: prostate-specific antigen.

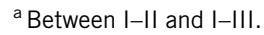

${ }^{b}$ Between I-III and II-III.

${ }^{\mathrm{c}}$ Between I-II, I-III and II-III.

informed written consent prior to their inclusion in the study. In this cross-sectional multicentre study, patients with similar profiles were recruited from six different institutions of the Aegean Region of Turkey. Patients with genital abnormalities, systemic diseases such as diabetes mellitus, thyroid diseases or neurological diseases or a history of transurethral resection or pelvic surgery and patients taking medication for LUTSs or ED were excluded.

\section{Patient evaluation}

After taking a detailed medical history and performing a digital rectal examination, serum prostate-specific antigen, testosterone levels and urinary flow rates were measured. Blood samples were drawn between 8:00 and 10:00 a.m. from overnight-fasting patients. For uroflowmetry studies, the patients were asked to urinate when they had a strong desire.

Patients were assessed based on the International Index of Erectile Function (IIEF) for ED, the International Prostate Symptom Score (IPSS) and the IPSS-Quality of Life (IPSS-QoL) for LUTSs and the Aging Males' Symptoms (AMS) scale for ageing male symptoms. The erectile function (EF) domain consists of questions 1-5 and 15, which aim to assess EF. ED was diagnosed when the total of the IIEF-EF domain scores was $<26$ points. ${ }^{7}$ The severity of ED according to the IIEF-EF score was classified into four categories as severe (1-10), moderate (11-16) or mild (17-25) ED or no ED (26-30). LUTSs were classified as mild (IPSS of $\leqslant 7$ ), moderate (IPSS of 8-19) or severe (IPSS of 20 or more). ${ }^{8}$ Patients with a total AMS score of at least 27 were considered to have SLOH symptoms. ${ }^{9}$ SLOH was classified as mild (AMS of 27-36), moderate (AMS of 37-49) or severe (AMS of 50 or more). The total AMS score and the domain scores, including the somatic (items: 1-2-3-4-5-9-10), sexual (items: 12-14-15-16-17) and psychological (items: 6-7-8-11-13) domains, were compared. At the end of data collection, the patients were divided into three groups according to the presence of LUTSs.

\section{Statistical analysis}

The patient characteristics, laboratory measurements and questionnaire scores, including the IIEF and AMS (total and subscales), were compared using one-way ANOVA. IIEF and AMS scores were compared between each group by a post-hoc Tukey test. The Pearson $\chi^{2}$ test was used to test the association of ED and SLOH with LUTSs, and crude odds ratios are given. The relationships between IPSS, AMS and IIEF scores were compared by the Pearson correlation test. Statistical significance was defined as $P<0.05$. The data were analysed using the Statistical Package for Social Sciences, version 11.0 (SPSS, Chicago, IL, USA), software program.

\section{RESULTS}

A total of 492 patients were included in the statistical analyses. Eight patients were not included in the statistical analyses due to lack of data. There was no significant difference between the mean ages of the moderate and severe LUTS groups, whereas the mild LUTS group was younger when compared with the other two groups (Table 1). The total PSA level was higher in patients with severe LUTSs $(P<0.001)$. The total testosterone level did not differ between groups.

Of the patients, $69.6 \%$ had ED. The presence of ED increased with increasing LUTS severity ( $\chi^{2}$ likelihood ratio: $6.9, P=0.032$ ). The prevalence of severe ED also increased with increasing LUTS severity (Figure 1).

SLOH (AMS > 27) was observed in $71.2 \%$ of the patients. When the groups were compared, the SLOH prevalence rates were $54.7 \%, 72.2 \%$ and $81.1 \%$ in the mild, moderate and severe LUTS groups, respectively. There were significant differences between the LUTS groups in both the total and somatic, sexual and psychological subgroup scores of the AMS (Table 2). The presence of SLOH increased with increasing LUTS severity ( $\chi^{2}$ likelihood ratio: $21.5, P<0.001$ ). The prevalence of severe SLOH also increased with increasing LUTS severity (Figure 2).

A correlation analysis revealed that all three questionnaire forms were correlated with each other (Table 3 ).

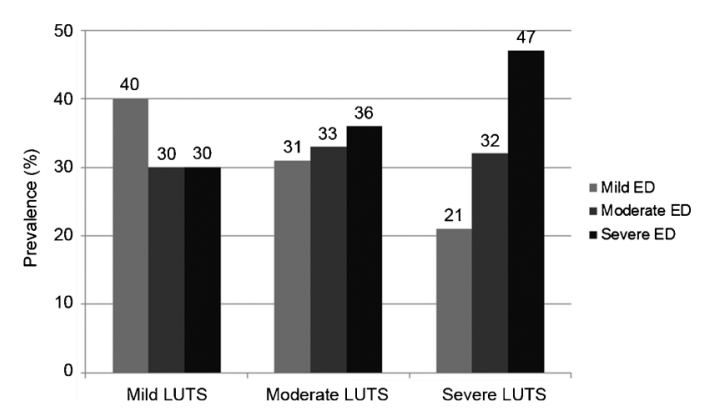

Figure 1 Relationship between LUTS severity with ED prevalence. The severity of ED is directly correlated with LUTS severity. ED, erectile dysfunction; LUTS, lower urinary tract symptoms. 
Table 2 Comparison of AMS scores between the three groups

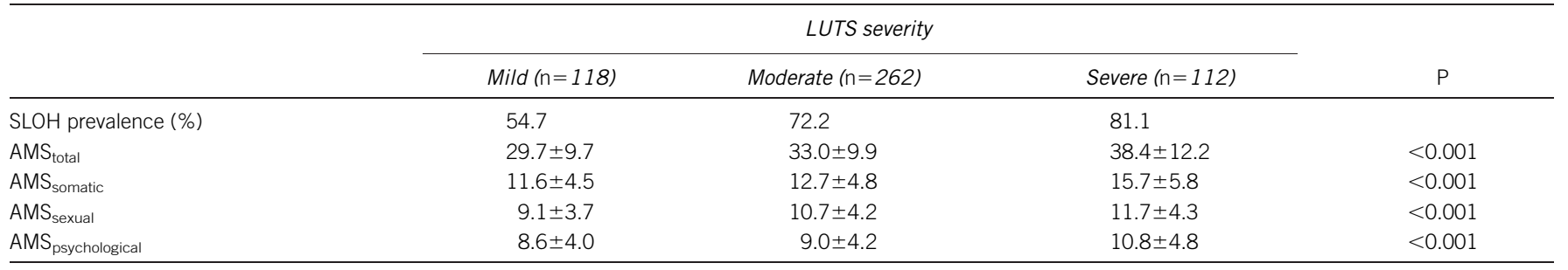

Abbreviations: AMS, Aging Males' Symptoms; LUTS, lower urinary tract symptom; SLOH, symptomatic late-onset hypogonadism.

\section{DISCUSSION}

Ageing is a known predominant risk factor for both male sexual dysfunction and LUTSs. The 'Massachusetts Male Aging Study' was the first to explore risk factors for ED in a large population. ${ }^{10}$ This study provided very valuable information about the basic risk factors for ED. According to this study, systemic diseases such as diabetes, hypertension, cardiovascular diseases and depression were age-independent risk factors for ED. However, LUTSs was not evaluated as a possible risk factor for ED in that study. In the population-based 'Cologne Male Survey', Braun et al. ${ }^{1}$ evaluated the relationship between LUTSs and ED with validated questionnaires by postal mail. They reported that sexual activity decreased and that ED prevalence increased with ageing. When they compared ED patients with nonED patients, the LUTS prevalence rates were $72.2 \%$ and $37.7 \%$ for the ED and non-ED groups, respectively. They proposed LUTSs as an independent risk factor for ED, as they demonstrated that patients with LUTSs had an odds ratio of 2.11 when compared with patients who did not have LUTSs.

One of the largest community-based studies, the 'Multinational Survey of the Aging Male' (MSAM-7), evaluated the relationship between LUTSs and sexual function in 12815 men aged 50-80 years in seven developed countries. ${ }^{2}$ They found that sexual activity decreased with increasing age and LUTS severity. LUTS severity was also highly predictive for all domains (EF, intercourse satisfaction, orgasmic function, sexual desire and overall satisfaction) of the IIEF questionnaire, and the IIEF domain scores decreased with increasing LUTS severity. They also demonstrated that LUTSs were a stronger predictor of male sexual dysfunction compared to other risk factors such as diabetes mellitus, hypertension, hyperlipidaemia and cardiac diseases. In the present study, the overall prevalence of ED was $69.6 \%$ when defined as an IIEF-EF score $<26$. This finding is similar to those of the studies referenced above. When we consider the effects of LUTS severity, we found that ED prevalence and severity increased with increasing LUTS severity in agreement with previous studies.

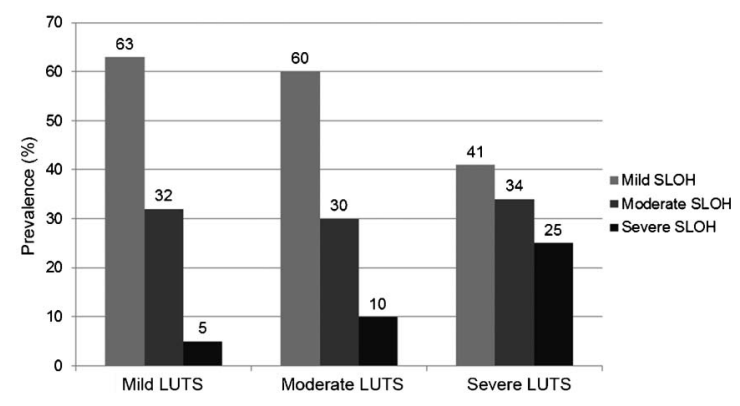

Figure 2 Relationship between LUTS severity and LOH symptoms. LOH symptom severity increases with increasing LUTS scores. LUTS, lower urinary tract symptoms; SLOH, symptomatic late-onset hypogonadism.
Additionally, when moderate and severe LUTS groups were compared, the IIEF-EF scores were significantly lower in the severe LUTS group, although no significant difference was found between the ages of these two groups. Our findings demonstrate that LUTS severity is an age-independent risk factor for ED.

$\mathrm{SLOH}$ is a condition characterized by various sexual, psychological and physiological symptoms affecting elderly men, primarily due to androgen levels decreasing with ageing. The 'International Society for the Study of the Aging Male' defined SLOH as a biochemical syndrome caused by a decrease in testosterone that occurs with ageing. ${ }^{11,12}$ Some intriguing studies have highlighted the role of sex steroids in the structure and function of the urinary tract, although the studies have primarily been performed in animals. ${ }^{13}$ These studies have identified androgen and oestrogen receptors in the urothelium and smooth muscle cells of the urethra and bladder in rats and rabbits as well as in the neurons in the autonomic ganglia of the prostatic plexus of male rats. ${ }^{14,15}$ Upon castration, the electrically evoked relaxation of the smooth muscle of the prostatic urethra was decreased. ${ }^{16}$ The Rhokinase activation/endothelin pathway may be involved in the increased smooth muscle activity found in LUTSs/benign prostate hyperplasia. ${ }^{17}$ Additionally, nitric oxide appears to have a smooth muscle relaxant effect on the urogenital organs. ${ }^{13}$

Few studies have analysed the relationship between circulating testosterone and LUTSs. Litman et al. ${ }^{18}$ reported a relationship between LUTSs and plasma total testosterone and bioavailable testosterone, but this relationship disappeared after adjusting for age. Rohrmann et al. ${ }^{19}$ evaluated the effect of circulating sex steroid hormones on LUTSs and concluded that androstanediol glucuronide, a metabolite of dihydrotestosterone and oestradiol were associated with an increased risk of LUTSs. Kim et al. ${ }^{20}$ investigated the relationship between sex hormone levels and LUTSs in patients with benign prostate hyperplasia, and they reported that endogenous testosterone may have a beneficial effect on lower urinary tract function and that a high frequency of nocturia may induce testosterone deficiency. In another study, Liao et al. ${ }^{21}$ showed that most IPSS items showed no significant association with serum sex hormone levels, except for nocturia, which showed a negative correlation with the serum testosterone level. In

Table 3 Correlations of questionnaire forms*

\begin{tabular}{lrcrr}
\hline & IPSS $_{\text {total }}$ & IPSS $_{\text {voiding }}$ & IPSS $_{\text {storage }}$ & \multicolumn{1}{l}{ IIEF-EF } \\
\hline AMS $_{\text {total }}$ & 0.306 & 0.267 & 0.267 & 0.417 \\
AMS $_{\text {somatic }}$ & 0.289 & 0.271 & 0.244 & -0.245 \\
AMS $_{\text {sexual }}$ & 0.238 & 0.211 & 0.210 & -0.569 \\
AMS $_{\text {psychological }}$ & 0.194 & 0.168 & 0.185 & -0.216 \\
IIEF-EF & -0.240 & -0.181 & -0.259 & \\
\hline
\end{tabular}

Abbreviations: AMS, Aging Males' Symptoms; IIEF-EF, International Index of Erectile Function-Erectile Function; IPSS, International Prostate Symptom Score. * $r$ values are provided in the table. All $P$ values were $<0.001$. 
contrast, Schatzl et al. ${ }^{22}$ reported that hypogonadism had no significant effect on symptom scores and flow rates in men with LUTSs. In another study, Miwa et al. ${ }^{23}$ reported that LUTSs were not associated with serum total testosterone and free testosterone levels, although the storage symptom scores of the IPSS were affected by the serum dehydroepiandrosterone sulphate level in older men. Interestingly, in our study, the mean serum testosterone levels did not differ between the mild, moderate and severe LUTS groups, despite symptomatic differences.

In the above-mentioned studies, only a hormonal evaluation was performed for SLOH, and a symptomatic assessment was not performed. In many studies, the symptomatic relationship between LUTSs and LOH has been analysed using testosterone replacement therapy (TRT). TRT is indicated in the presence of both clinical symptoms suggestive of hormone deficiency and a decreased testosterone level. ${ }^{12,24}$ Although several testosterone formulations are available, topical testosterone gels and long-term depot injections are the most commonly used treatment options due to their favourable pharmacokinetic profiles, characterized by relatively constant plasma levels, avoiding wide fluctuations and minimal side effects. ${ }^{25}$ Amano et al. ${ }^{26}$ evaluated the efficacy of TRT using testosterone ointment (Glowmin) for LUTSs in LOH patients. They reported that serum free testosterone levels and the scores for the four parameters of the AMS, six of eight domains in the 36-Item Short Form Health Survey (SF-36), the IIEF-5 and the total IPSS improved significantly after 3 months of TRT. They demonstrated that the voiding disturbance improved more than the storage disturbance. Karazindiyanoglu and Cayan $^{27}$ reported that TRT with transdermal testosterone $50-100 \mathrm{mg}$ of gel per day for 1 year significantly improved bladder function by increasing bladder capacity and compliance and decreasing detrusor pressure at maximal flow in 25 patients with SLOH. Kalinchenko et al. ${ }^{28}$ showed that TRT with either daily testosterone gel (50 mg) application or injections of $1000 \mathrm{mg}$ of testosterone undecanoate also improved many parameters, including plasma testosterone levels, the IPSS, the AMS scale and the IIEF, in LOH men. Recently, Shigehara et al. ${ }^{29}$ reported a randomized controlled study evaluating the effects of ART on LUTSs in 52 hypogonadal men with mild BPH. Fifty-two patients were randomly assigned to receive testosterone (ART group 26 patients) as $250 \mathrm{mg}$ of testosterone enanthate every 4 weeks or to the untreated control group (26 patients), and the IPSS, UFM data and PVR were compared at baseline and 12 months after treatment. At the 12-month visit, the IPSS showed a significant decrease compared with baseline, and the maximum flow rate and voided volume showed significant improvements in the TRT group; no significant improvements were observed in the controls. However, Takao et al. ${ }^{30}$ reported that the efficacy of TRT by testosterone or HCG injection was questionable. Additionally, recent guidelines on TRT note that the diagnosis or suspicion of prostate cancer and severe LUTSs due to BPH are relative contraindications to TRT. ${ }^{31,32}$

To the best of our knowledge, our study is the first in the literature to compare AMS scores with LUTS severity. Atan et al. ${ }^{33}$ found no significant correlation between total AMS scores and ageing in their study in which they evaluated the effects of ageing on LUTSs, EF and SLOH. However, they did not report the relationship between LUTS severity and SLOH symptoms. In a recent study, the relationships between LUTSs, ED and depression in Japanese patients with SLOH were investigated. That study revealed that moderate and severe ED was correlated with depression, whereas LUTSs were not related to depression in Japanese SLOH patients. ${ }^{34}$ We found the prevalence of SLOH to be $71.2 \%$ in men with LUTSs in the present study. We also determined that the SLOH prevalence increased with increasing LUTS severity. When the AMS subscales in three groups were examined, we also determined that LUTS severity has a detrimental effect on all subscales of the AMS and the total AMS score. When patients with SLOH were compared with patients without SLOH, the total IPSS and the IPSS voiding and storage symptom scores were all significantly higher in patients with SLOH.

There are some limitations to our study. First, serum-free and bioavailable $\mathrm{T}$ levels were not measured due to the multi-institutional nature of the study, although they may differ between groups. Second, we evaluated only patients presenting with urinary symptoms to urology clinics, and our study does not refer to the relationships between LUTSs, ED and SLOH in the general population.

\section{CONCLUSIONS}

In this multicentre, cross-sectional analysis of LUTSs, ED and SLOH, LUTS severity was an age-independent risk factor for ED and SLOH. Patients with severe LUTSs were more likely to have ED and SLOH compared with patients with mild and moderate LUTS. Additionally, the prevalence of severe ED and SLOH increased with increasing LUTS severity. Furthermore, validated forms of ED, LUTSs and SLOH were associated with each other in patients with LUTS. We also suggest that LUTS severity and SLOH symptoms are closely associated, although this hypothesis requires etiological and biological clarification in future studies with large sample sizes.

\section{COMPETING FINANCIAL INTERESTS}

The authors have no competing financial interests.

\section{AUTHOR CONTRIBUTIONS}

OB and OD made contributions to the study conception and design, data acquisition, analysis and interpretation, and the drafting and revising of the manuscript. DB made contributions to the study conception and design, data acquisition, and the drafting and revising of the manuscript. OU, AS, BO, AP, TT, BG, EC, AB, HE and AE made contributions to the study conception and design, data acquisition and the revising of the manuscript.

All authors read and approved the final manuscript.

1 Braun M, Wassmer G, Klotz T, Reifenrath B, Mathers M et al. Epidemiology of erectile dysfunction: results of the 'Cologne male survey'. Int J Impot Res 2000; 12: 305-11. 2 Rosen R, Altwein J, Boyle P, Kirby RS, Lukacs B et al. Lower urinary tract symptoms and male sexual dysfunction: the multinational survey of the aging male (MSAM-7). Eur Urol 2003; 44: 637-49.

3 Vallancien G, Emberton M, Harving N, van Moorselaar RJ; Alf-One Study Group. Sexual dysfunction in 1.274 European men suffering from lower urinary tract symptoms. J Urol 2003; 169: 2257-61.

4 Li MK, Garcia LA, Rosen R. Lower urinary tract symptoms and male sexual dysfunction in Asia: a survey of aging men from 5 Asian countries. BJU Int 2005; 96: 1339-54.

5 McVary K. Lower urinary tract symptoms and sexual dysfunction: epidemiology and pathophysiology. BJU Int 2006; 97(Suppl 2): 23-8.

6 Araujo AB, O'Donnell AB, Brambilla DJ, Simpson WB, Lonqcope J et al. Prevalence and incidence of androgen deficiency in middle-aged and older man: estimates from the Massachusetts Male Aging Study. J Clin Endocrinol Metab 2004; 89: 5920-6.

7 Rosen RC, Riley A, Wagner G, Osterloh IH, Kirkpatrick J et al. The international index of erectile function (IIEF): a multidimensional scale for assessment of erectile dysfunction. Urology 1997; 49: 822-30.

8 Barry MJ, Fowler FJ Jr, O'Leary MP, Bruskewitz RC, Holtgrewe HL et al. The American Urological Association symptom index for benign prostatic hyperplasia. The Measurement Committee of the American Urological Association. J Urol 1992; 148: 1549-57.

9 Heinemann LA. Aging Males' Symptoms scale: a standardized instrument for the practice. J Endocrinol Invest 2005; 28: 34-8.

10 Feldman HA, Goldstein I, Hatzichristou DG, Krane RJ, McKinlay JB. Impotence and its medical and psychosocial correlates: results of the Massachusetts Male Aging Study. J Urol 1994; 151: 54-61. 
11 Morales A, Lunenfeld B. Investigation, treatment and monitoring of late-onset hypogonadism in males. Official recommendations of ISSAM. International Society for the Study of the Aging Male. Aging Male 2002; 5: 74-86.

12 Nieschlag E, Swerdloff R, Behre HM, Gooren LJ, Kaufman JM et al. Investigation, treatment and monitoring of late-onset hypogonadism in males: ISA, ISSAM, and EAU recommendations. Eur Urol 2005; 48: 1-4.

13 Pradidarcheep W. Lower urinary tract symptoms and its potential relation with lateonset hpogonadism. Aging Male 2008; 11: 51-5.

14 Rosenzweig BA, Bolina PS, Birch L, Moran C, Marcovici I et al. Location and concentration of estrogen, progesterone, and androgen receptors in the bladder and urethra of the rabbit. Neurourol Urodyn 1995; 14: 87-96.

15 Salmi S, Santti R, Gustafsson JA, Makela S. Co-localization of androgen receptor with estrogen receptor beta in the lower urinary tract of the male rat. J Urol 2001; 166: 674-7.

16 Holmquist F, Persson K, Bodker A, Anderson KE. Some pre- and post-junctiona effects of castration in rabbit isolated corpus cavernosum and urethra. $J$ Urol 1994; 152: $1011-6$

17 Peters SL, Schmidt M, Michel MC. Rho kinase: a target for treating urinary bladder dysfunction? Trends Pharmacol Sci 2006; 27: 492-7.

18 Litman HJ, Bhasin S, O'Leary MP, Link CL, McKinlay JB. An investigation of the relationship between sex-steroid levels and urological symptoms: results from the Boston Area Community Health Survey. BJU Int 2007; 100: 321-6.

19 Rohrmann S, Nelson WG, Rifai N, Kanarek N, Basaria S et al. Serum sex steroid hormones and lower urinary tract symptoms in third National Health and Nutrition Examination Survey (NHANES III). Urology 2007; 69: 708-13.

20 Kim MK, Zhao C, Kim SD, Kim DG, Park JK. Relationship of sex hormones and nocturia in lower urinary tract symptoms induced by benign prostatic hyperplasia. Aging Male 2012; 15: 90-5.

21 Liao $\mathrm{CH}$, Chiang HS, Yu HJ. Serum testosterone levels significantly correlate with nocturia in men aged 40-79 years. Urology 2011; 78: 631-5.

22 Schatzl G, Brössner C, Schmid S, Kuqler W, Roehrich M et al. Endocrine status in elderly men with lower urinary tract symptoms: correlation of age, hormonal status and lower urinary tract function. The Prostate Study Group of the Austrian Society of Urology. Urology 2000; 55: 397-402.
23 Miwa Y, Kaneda T, Yokoyama O. Association between lower urinary tract symptoms and serum sex hormones in men. Urology 2008; 72: 552-5.

24 Vermeulen A. Androgen replacement therapy in the aging male-a critical evaluation. $J$ Clin Endocrinol Metab 2001; 86: 2380-90.

25 Hatzimouratidis $\mathrm{H}$, Hatzichristou D. Testosterone and erectile function: an unresolved enigma. Eur Urol 2007; 52: 26-8.

26 Amano T, Imao T, Takemae K, Iwamoto T, Nakanome M. Testosterone replacement therapy by testosterone ointment relieves lower urinary tract symptoms in late onset hypogonadism patients. Aging Male 2010; 13: 242-6.

27 Karazindiyanoglu S, Cayan S. The effects of testosterone therapy on lower urinary tract symptoms/bladder and sexual functions in men with symptomatic late-onset hypogonadism. Aging Male 2008; 11: 146-9.

28 Kalinchenko S, Vishnevsky EL, Koval AN, Mskhalaya GJ, Saad F. Benefical effects of testosterone administration on symptoms of the lower urinary tract in men with lateonset hypogonadism: a pilot study. Aging Male 2008; 11: 57-61.

29 Shigehara K, Sugimoto K, Konaka H, lijima M, Fukushima M et al. Androgen replacement therapy contributes to improving lower urinary tract symptoms in patients with hypogonadism and benign prostate hypertrophy: a randomised controlled study. Aging Male 2011; 14: 53-8.

30 Takao T, Tsujimura A, Nakayama J, Matsuoka Y, Miyagawa Y et al. Lower urinary tract symptoms after hormone replacement therapy in Japanese patients with late-onset hypogonadism: a preliminary report. Int J Urol 2009; 16: 212-4.

31 Bhasin S, Cunningham GR, Hayes FJ, Matsumoto AM, Snyder PJ et al. Testosterone therapy in men with androgen deficiency syndromes: an Endocrine Society clinical practice guideline. J Clin Endocrinol Metab 2010; 95: 2536-59.

32 Wang C, Nieschlag E, Swerdloff R, Beher HM, Hellstrom WJ et al. Investigation, treatment and monitoring of late-onset hypogonadism in males. Int J Androl 2009 32: $1-10$.

33 Atan A, Basar MM, Tuncel A, Mert C, Aslan Y. Is there a relationship among age International Index of Erectile Function, International Prostate Symptom Score, and Aging Males' Symptoms score? Int Urol Nephrol 2007; 39: 215-22.

34 Takao T, Tsujimura A, Okuda H, Yamamoto K, Fukuhara S et al. Lower urinary tract symptoms and erectile dysfunction associated with depression among Japanese patients with late-onset hypogonadism symptoms. Aging Male 2011; 14: 110-4. 